\title{
Bragg Spectroscopy of Cold Atomic Fermi Gases
}

\author{
G. M. Bruun ${ }^{1}$ and Gordon Baym ${ }^{2,3}$ \\ ${ }^{1}$ Niels Bohr Institute, Blegdamsvej 17, 2100 Copenhagen, Denmark \\ ${ }^{2}$ NORDITA, Blegdamsvej 17, 2100 Copenhagen, Denmark \\ ${ }^{3}$ Department of Physics, University of Illinois, 1110 W. Green St., Urbana, IL 61801
}

(Dated: January 23, 2018)

\begin{abstract}
We propose a Bragg spectroscopy experiment to measure the onset of superfluid pairing in ultracold trapped Fermi gases. In particular, we study two component Fermi gases in the weak coupling BCS and BEC limits as well as in the strong coupling unitarity limit. The low temperature Bragg spectrum exhibits a gap directly related to the pair-breaking energy. Furthermore, the Bragg spectrum has a large maximum just below the critical temperature when the gas is superfluid in the BCS limit. In the unitarity regime, we show how the pseudogap in the normal phase leads to a significant suppression of the low frequency Bragg spectrum.
\end{abstract}

\section{INTRODUCTION}

Bragg spectroscopy, using two laser beams, has proven to be a very successful tool for probing the structure factor of trapped atomic BEC's [1]. In this paper, we examine the analogous experiment on two-component Fermi gases in both the normal and superfluid phases. We calculate the Bragg scattering rate for varying interaction strengths in both the weak coupling BCS and BEC limits and in the strong coupling unitarity limit. Previously, we proposed measuring the inelastic (Stokes/antiStokes) scattering of an off-resonant laser beam on a twocomponent Fermi gas [2]. In the BCS limit, the intensity of the scattering in this Stokes experiment exhibits a large maximum just below the critical temperature, $T_{c}$, at which the gas becomes superfluid. This effect is the light scattering analog of the Hebel-Slichter effect in conventional superconductors, a hallmark experimental test of BCS theory [3]. Here, we demonstrate that the same effect can be observed in a Bragg scattering experiment. We furthermore show that the pairing induced by the interactions can be readily detected in the Bragg spectrum. In the strong coupling regime, we discuss how the presence of a pseudogap in the normal phase has significant effects on the Bragg spectrum. The advantage of the Bragg experiment as compared to the Stokes experiment is that one varies in a controlled way the energy and momentum imparted to the atoms. Compared with recent radio frequency (rf) experiments which involve a third unoccupied hyperfine state [4], the Bragg experiment involves only the two hyperfine states already present in the trap. The Bragg experiment, avoiding complications due to non-trivial interaction effects between the third hyperfine state present in the rf experiment [5] and the trapped gas, is thus simpler to interpret.

\section{BRAGG SCATTERING}

We consider a homogeneous gas of fermionic atoms occupying two hyperfine states, denoted by $|\uparrow\rangle$ and $|\downarrow\rangle$. We assume that the state $|\downarrow\rangle$ has energy $\omega_{\text {hf }}(\hbar=1$ and

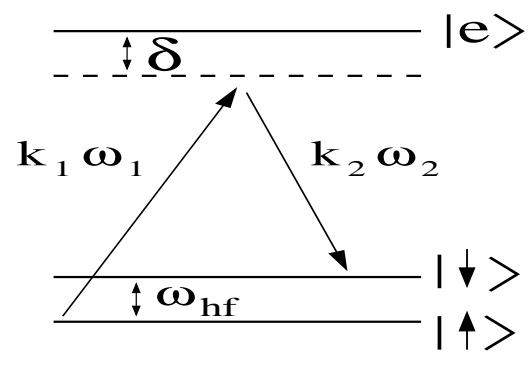

FIG. 1: Schematic atomic level diagram for stimulated Bragg scattering leading to a "spin" flip.

$k_{B}=1$ in this paper) above the state $|\uparrow\rangle$, and that the population of the two states is equal, $N_{\uparrow}=N_{\downarrow}=N / 2$. The two atomic states interact with a coupling which can be characterized by a scattering length $a$ in a vacuum. We ignore the interaction between atoms in the same hyperfine state since $s$-wave scattering is suppressed between two identical fermions.

Bragg scattering is realized by a pair of laser beams with wave vectors $\mathbf{k}_{1}$ and $\mathbf{k}_{2}$ and frequencies $\omega_{1}$ and $\omega_{2}$ illuminating the gas. An atom absorbing a photon from beam 1 and emitting a photon in beam 2 changes its energy-momentum by $\omega_{1}-\omega_{2}, \mathbf{k}_{1}-\mathbf{k}_{2}$. Bragg scattering can be used to probe both the density and spin correlation functions. Pairing is predicted to have several effects on the frequency and momentum dependence of these correlation functions for $T=066,7,8]$. To probe the spin-flip correlation function, one tunes the two laser frequencies so that $\omega_{1}-\omega_{2} \sim \omega_{\text {hf }}$ as illustrated in Fig. 1]

If the detuning, $\delta$, from the optical transition to or from the upper level $|e\rangle$ is large, population of the upper level can be neglected, and the effective light matter Hamiltonian describing spin-flip Bragg scattering can be written as

$$
H_{\mathrm{B}}=\int d^{3} r\left[\mathcal{I}(\mathbf{r}, t) \psi_{\downarrow}^{\dagger}(\mathbf{r}, t) \psi_{\uparrow}(\mathbf{r}, t)+\text { h.c. }\right],
$$

where $\mathcal{I}(\mathbf{r}, t)=e^{-i\left(\omega_{1}-\omega_{2}\right) t} \mathcal{I}(\mathbf{r})$, and $\mathcal{I}(\mathbf{r})$ describes the spatial profile of the laser beams and the dependence on the atomic states involved and their coupling to the 
electromagnetic field [9]. We neglect finite waist effects and take $\mathcal{I}(\mathbf{r})=\mathcal{I} e^{i \mathbf{q} \cdot \mathbf{r}}$ with $\mathbf{q}=\mathbf{k}_{1}-\mathbf{k}_{2}$.

To measure the effects of pairing we focus on the spinflip rate $\left\langle\dot{N}_{\downarrow}\right\rangle$ induced by the Bragg lasers, where $N_{\downarrow}$ is here the number of particles in the level $|\downarrow\rangle$, and $\langle\ldots\rangle$ denotes a thermal average. The Heisenberg equation of motion for the operator $N_{\downarrow}$ is

$$
i \dot{N}_{\downarrow}=\left[N_{\downarrow}, H_{\mathrm{B}}\right]=\int d^{3} r\left[\mathcal{I}(\mathbf{r}, t) \psi_{\downarrow}^{\dagger}(\mathbf{r}, t) \psi_{\uparrow}(\mathbf{r}, t)-\text { h.c. }\right] .
$$

For sufficiently weak laser beams, $\left\langle\dot{N}_{\downarrow}\right\rangle$ can by calculated by linear response theory:

$$
\begin{gathered}
\left\langle\dot{N}_{\downarrow}\right\rangle=i \mathcal{I}^{2} \int \frac{d \omega^{\prime}}{2 \pi}\left(\frac{\left\langle\left[\psi_{\uparrow}^{\dagger} \psi_{\downarrow}, \psi_{\downarrow}^{\dagger} \psi_{\uparrow}\right]\right\rangle(\mathbf{q}, \omega)}{\omega-\omega^{\prime}}-\text { c.c. }\right) \\
=-2 \mathcal{I}^{2} \operatorname{Im} \mathcal{D}(\mathbf{q}, \omega+i \eta),
\end{gathered}
$$

with $\omega=\omega_{1}-\omega_{2}-\omega_{\mathrm{hf}}$. Bragg scattering thus probes the Fourier transform of the spin-flip correlation function $\left\langle\left[\psi_{\uparrow}^{\dagger}(\mathbf{r}, t) \psi_{\downarrow}(\mathbf{r}, t), \psi_{\downarrow}^{\dagger}\left(\mathbf{r}^{\prime}, t^{\prime}\right) \psi_{\uparrow}\left(\mathbf{r}^{\prime}, t^{\prime}\right)\right]\right\rangle$. The response of the gas is usually detected experimentally by measuring the total momentum imparted on the gas [1]; the rate of momentum transfer is straightforwardly related to the scattering rate by

$$
\dot{\mathbf{P}}=\mathbf{q} \dot{N}_{\downarrow}
$$

where $\mathbf{P}=\mathbf{P}_{\downarrow}+\mathbf{P}_{\uparrow}$ is the total momentum of the gas in the two hyperfine states.

\section{WEAK COUPLING BCS LIMIT}

In the weak coupling limit $a \rightarrow 0^{-}$, the gas can be described by BCS theory. The correlation function in (3) is then a sum of contributions from normal and anomalous Green's functions [10]. We obtain after some algebra,

$$
\begin{gathered}
\mathcal{D}_{0}(q, z)=\int \frac{d^{3} k}{(2 \pi)^{3}}\left[\left(u u^{\prime}+v v^{\prime}\right)^{2} \frac{f-f^{\prime}}{z+E-E^{\prime}}+\right. \\
\left.\frac{1}{2}\left(u v^{\prime}-v u^{\prime}\right)^{2}\left(\frac{\left(1-f-f^{\prime}\right)}{z-E-E^{\prime}}+\frac{\left(f+f^{\prime}-1\right)}{z+E+E^{\prime}}\right)\right],
\end{gathered}
$$

with $z=\omega+i \eta$. Here, $u=u_{k}$ and $u^{\prime}=u_{\mathbf{k}+\mathbf{q}}$, etc., with $u_{k}^{2}=\left(1+\xi_{k} / E_{k}\right) / 2$ and $v_{k}^{2}=1-u_{k}^{2}$ as usual in BCS theory. The quasiparticle energies are $E=E_{k}=\sqrt{\xi_{k}^{2}+\Delta^{2}}$ and $E^{\prime}=E_{\mathbf{k}+\mathbf{q}}$ with $\xi_{k}=\epsilon_{k}-\mu$, where $\epsilon_{k}=k^{2} / 2 m$, $\mu$ is the chemical potential, and $\Delta$ the BCS pairing gap. The Fermi functions are $f=f(E)$ and $f^{\prime}=f\left(E^{\prime}\right)$, with $f(x)=\left(e^{x / T}+1\right)^{-1}$. The first term of (5), describes scattering of quasiparticles while the last two terms describe the creation and annihilation of two quasiparticles - pair breaking processes.

For an ideal gas in the normal phase, (5) yields in the limit $\omega \ll \epsilon_{F}, T \ll T_{F}$ ( $T_{F}$ is the Fermi temperature), and $\omega \ll q k_{F} / m$,

$$
\operatorname{Im} \mathcal{D}_{0}(q, \omega)=-\frac{3 \pi}{16} \frac{n k_{F} \omega}{q \epsilon_{F}^{2}},
$$

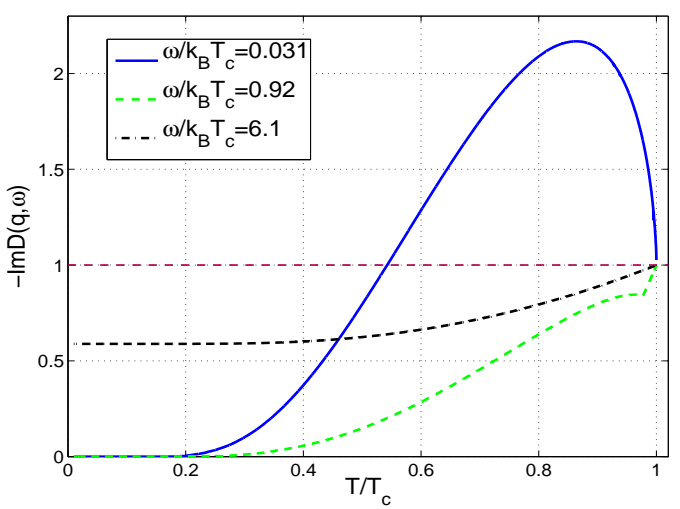

FIG. 2: The scattering rate $-\operatorname{Im} D(q, \omega)$ in units of the scattering rate in the normal phase vs. $T$ for representative $\omega$. (color online).

where $\epsilon_{F}$ is the Fermi energy, $n=k_{F}^{3} / 3 \pi^{2}$ is the total density of the gas, and $k_{F}$ is the Fermi momentum.

The spin flip induced by Bragg scattering can excite collective spin waves. These modes are undamped in the normal phase for an attractive interaction [11]. For a repulsive interaction in the normal phase, the spin waves are damped and they change the spectral shape of the particle-hole continuum. The single particle approximation (5) does not include such collective modes. In order to include them, we sum bubbles to obtain the random phase (RPA) approximation [10],

$$
\mathcal{D}_{\mathrm{RPA}}=\frac{\mathcal{D}_{0}}{1+\Gamma \mathcal{D}_{0}}
$$

with $\Gamma=4 \pi a / m$ the scattering matrix for $k_{F} a \ll 1$. Note the + sign in the denominator due to the spin flip.

\section{A. Results}

We first consider the temperature dependence of the scattering rate. An attractive feature of the Stokes experiment is that one can observe a strikingly large maximum in the scattered signal just below the critical temperature $T_{c}$ [2]. We now demonstrate that the same effect is present for the Bragg scattering experiment in the experimentally realizable parameter range, $\omega \ll \Delta$, $\omega<q k_{F} / m$, and $q<2 k_{F}$.

Figure 2 shows the scattering rate (3) as a function of $T$ in the weak coupling limit, in units of the normal phase rate, which is constant for $T \ll T_{F}$. For illustration, we have taken $k_{F} a=-0.3$ and $q=k_{F}$. Solving the BCS equations as a function of $T$, we obtain the scattering rate from Eqs. (5) and (77). The critical temperature is $T_{c}=0.0033 \epsilon_{F}$. We plot the results for frequency differences between the two laser beams $\omega=0.031 T_{c}, 0.92 T_{c}$, and $6.1 T_{c}$. The numerical normal phase results agree with (6), confirming the numerical accuracy of the calculations. 
As Fig. 2 shows, the scattering rate in the superfluid state has a large maximum below $T_{c}$ for $\omega \ll T_{c}$. This maximum is due to a large quasiparticle scattering rate arising from the increased density of states at the Fermi energy in the superfluid phase just below $T_{c}$, $N(E)=\left(p_{F} / 2 \pi^{2}\right) E_{k} / \xi_{k}$. For $\omega \ll T_{c}$, both scattering states $\mathbf{k}$ and $\mathbf{k}+\mathbf{q}$ in Eq. (5) can be in the region around the Fermi surface of a large density of states yielding an increased scattering rate. The maximum in the scattering rate depends crucially on the coherence factors for quasiparticle scattering in (5) adding, viz., $u u^{\prime}+v v^{\prime} \sim 1$, for a spin flip. For Bragg scattering into the same hyperfine state, the coherence factor is $u u^{\prime}-v v^{\prime} \sim 0$ at the Fermi surface and the increased density of states would not lead to a maximum in the scattering rate. For $T \rightarrow 0$, the scattering rate is suppressed by the increasing gap $\Delta(T)$ since the density of quasiparticles available for scattering scales as $\exp (-\Delta / T)$. For $\omega<2 \Delta$ the lasers cannot break Cooper pairs.

The physical reason for the coherence factors adding $\left(u u^{\prime}+v v^{\prime} \sim 1\right)$ for spin-flip scattering is that the quasiparticles carry the same spin in the superfluid as in the normal phase. On the other hand, in scattering without spin flip, which couples to the particle density, the particle number carried by the quasiparticles is suppressed at the Fermi surface, since a quasiparticle is a superposition of a hole and a particle. In this case the coherence factors subtract $\left(u u^{\prime}-v v^{\prime}\right)$.

For increasing $\omega$, the peak in the rate below $T_{c}$ decreases and eventually disappears for $\omega \sim T_{c}$. From Fig. 22 we see that the scattering rate in the superfluid state is always smaller than in the normal state when $\omega \gtrsim T_{c}$ (green dashed and black dash-dotted lines). This is because both scattering states cannot be in the region around the Fermi surface where the single particle density of states is increased. Bragg scattering does not probe the increased density of states for the superfluid state for higher frequency as effectively as for $\omega \ll T_{c}$, and the resulting rate is decreased compared to the normal state. Note that the kink in scattering rate just below $T_{c}$ for $\omega=0.92 T_{c}$, at which point $2 \Delta(T)=\omega$, is physical. Below this temperature, the gap is too large to allow the creation of two quasiparticles and the second term in (5) vanishes.

Finally, Fig. 2 for $\omega=6.1 T_{c}$ illustrates the case of large energy transfer, $\omega>2 \Delta(T=0)$. Contrary to the case $\omega<2 \Delta(T=0)$, the rate is finite for $T=0$ as the energy transfer is large enought to break pairs and the second term in (15) is finite. For $\omega / T_{c} \rightarrow \infty$, the scattering rate approaches that of the normal state, as expected.

We conclude that there is a peak in the scattering rate for $T<T_{c}$ and $\omega \ll T_{c}$. Also, the peak disappears unless $\omega<q k_{F} / m$ and $q<2 k_{F}$; otherwise, both scattering states cannot be in the region of increased density of states.

We now consider the frequency dependence of the Bragg scattering rate. Figure 3 shows the rate from (7) as a function of $\omega$ for the gas in the weak coupling limit

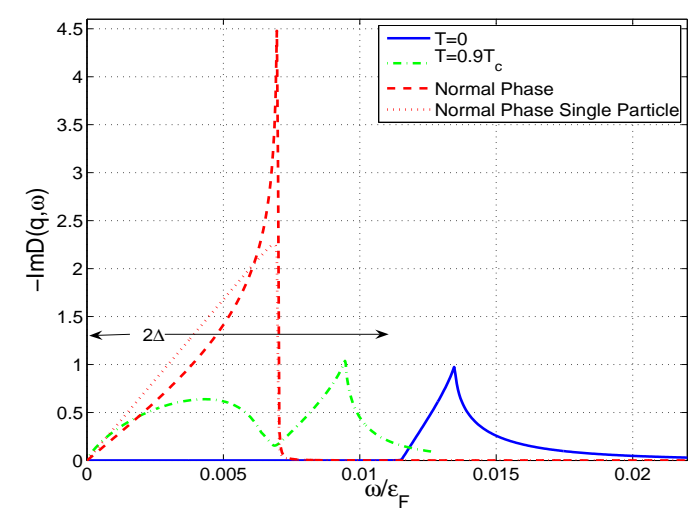

FIG. 3: The scattering rate $-\operatorname{Im} D(q, \omega)$ in units of $n / 2 \epsilon_{F}$ as a function of $\omega$ for $k_{F} a=-0.3$ and various temperatures (color online).

with $k_{F} a=-0.3$ and $q / k_{F}=0.0035$. The scattering rate is depicted for various temperatures below and just above $T_{c}$ where the gas is normal. For comparison, we also plot the result of the single particle approximation (5]) in the normal phase.

The large peak in the normal phase response just above the single particle continuum arises from the spin wave present above the particle-hole continuum for an attractive interaction. The finite width of the peak is an artifact of our having included a small imaginary part in the frequency $\omega$ for computational reasons. We see from Fig. 3 that when the gas becomes superfluid, the spin wave vanishes. This is because only potential flow, i.e., density fluctuation modes, is possible in the superfluid [12]. Other modes such as spin waves require a normal component to be present. In a trapped system this effect leads to the damping of the spin-dipole mode in the superfluid phase [13]. In the superfluid phase, the particle-hole spectrum disappears and is replaced by a low frequency gap, which reflects the pair breaking energy described by the second term in (5). At zero temperature the gas does not respont for $\omega<2 \Delta$, the minimum energy required to break a pair.

The pairing gap can thus be detected directly as a gap of $2 \Delta$ in the Bragg spectrum. By contrast, in the rf experiment of 4$]$ the shift in the spectrum scales as $\Delta^{2} / \epsilon_{F}$, since one transfers particles to a third hyperfine state which in general interacts differently with the two paired hyperfine states [5].

\section{WEAK COUPLING BEC LIMIT}

We now consider Bragg scattering in the weak coupling BEC regime $k_{F} a \rightarrow 0^{+}$. In this limit, the molecules are tightly bound with a radius much smaller than the average interparticle distance, and are weakly interacting both with each other and with unbound atoms 14]. The system can therefore to a good approximation be 
regarded as an ideal gas of point molecules and atoms in thermal equilibrium. The molecules Bose condense below the critical temperature $T_{c}=0.218 T_{F}$. The Bragg scattering induced by (11) then either breaks a molecule by flipping the spin of a bound $\uparrow$ atom, thereby creating two free $\downarrow$ atoms, or it simply flips the spin of a free $\uparrow$ atom. The Fermi Golden Rule result for Bragg scattering rate into the free atomic $\downarrow$ states for such a system is then a sum of three terms,

$$
\dot{n}_{\downarrow}=\mathcal{I}^{2}\left(\Gamma_{\text {atom }}+\Gamma_{\mathrm{BEC}}+\Gamma_{\mathrm{Mol}}\right) .
$$

The scattering from the unbound atoms is given by

$$
\Gamma_{\text {atom }}=\int \frac{d^{3} k}{(2 \pi)^{3}}\left(f-f^{\prime}\right) 2 \pi \delta\left(\omega+\epsilon-\epsilon^{\prime}\right),
$$

where $\epsilon=\epsilon_{k}, \epsilon^{\prime}=\epsilon_{\mathbf{k}+\mathbf{q}}, f=f(\xi)$, and $f^{\prime}=f\left(\xi^{\prime}\right)$. The rate from a BEC of molecules with density $n_{0}$ is

$\Gamma_{\mathrm{BEC}}=n_{0} \int \frac{d^{3} k}{(2 \pi)^{3}}|M|^{2}\left(1-f-f^{\prime}\right) 2 \pi \delta\left(\omega+E_{b}-\epsilon-\epsilon^{\prime}\right)$,

with $E_{b}=-\hbar^{2} / m a^{2}$ the energy of a molecule with zero momentum relative to the bottom of the free particle continuum of a pair of $\uparrow$ and $\downarrow$ atoms, and $M=M(\mathbf{k}, \mathbf{q})$ the matrix element of (1) (excluding $\mathcal{I}$ ) between a molecule at rest and a pair of free $\downarrow$ atoms with momenta $\mathbf{k}+\mathbf{q}$ and $-\mathbf{k}$. The scattering rate from thermal molecules is

$$
\begin{gathered}
\Gamma_{\mathrm{Mol}}=\int \frac{d^{3} p}{(2 \pi)^{3}} \int \frac{d^{3} k}{(2 \pi)^{3}}|M|^{2} \\
{\left[n_{B}\left(\epsilon_{\mathrm{mol}}\right)\left(1-f-f^{\prime}\right)-f f^{\prime}\right] 2 \pi \delta\left(\omega+E_{b}+\frac{p^{2}}{4 m}-\epsilon-\epsilon^{\prime}\right),}
\end{gathered}
$$

with $n_{B}(x)=\left(e^{x / T}-1\right)^{-1}$ the Bose distribution, and $\epsilon_{\mathrm{mol}}(p)=E_{b}+p^{2} / 4 m-2 \mu$ the energy of molecules with momentum $p$. The molecule breaks into two $\downarrow$ atoms with momenta $\mathbf{k}+\mathbf{q}+\mathbf{p} / 2$ and $\mathbf{p} / 2-\mathbf{k}$ and energies $\epsilon=\epsilon_{\mathbf{p} / 2-\mathbf{k}}$ and $\epsilon^{\prime}=\epsilon_{\mathbf{k}+\mathbf{q}+\mathbf{p} / 2}$. The matrix element in (11) is, from Galilean invariance, independent of $\mathbf{p}$.

To proceed, we model the molecule-free atom pair matrix element $M=M(\mathbf{k}, \mathbf{q})$ as follows. We write state of a molecule at rest as $|\phi\rangle=\sum_{\mathbf{k}} \phi_{k} c_{\mathbf{k} \uparrow}^{\dagger} c_{-\mathbf{k} \downarrow}^{\dagger}|0\rangle$, with $|0\rangle$ the vacuum and $c_{\mathbf{k} \sigma}^{\dagger}$ creating an atom with momentum $\mathbf{k}$ in hyperfine state $\sigma$. The Fourier transform of the molecular wave function is $\phi_{k}$. The spin-flip process illustrated in Fig. 1 breaking the molecule and adding momentum $\mathbf{q}$ creating two free $\downarrow$ atoms with momenta $\mathbf{k}+\mathbf{q}$ and $-\mathbf{k}$ corresponds to $c_{\mathbf{k}+\mathbf{q} \downarrow}^{\dagger} c_{\mathbf{k} \uparrow}|\phi\rangle=\phi_{k} c_{\mathbf{k}+\mathbf{q} \downarrow}^{\dagger} c_{-\mathbf{k} \downarrow}^{\dagger}|0\rangle$. However, the process $c_{-\mathbf{k} \downarrow}^{\dagger} c_{-\mathbf{k}-\mathbf{q} \uparrow}|\phi\rangle=-\phi_{|\mathbf{k}+\mathbf{q}|} c_{\mathbf{k}+\mathbf{q} \downarrow}^{\dagger} c_{-\mathbf{k} \downarrow}^{\dagger}|0\rangle$ connects to the same final state, and the two matrix element must be added coherently in the Golden Rule expression. We thus find the matrix element $|M(\mathbf{k}, \mathbf{q})|^{2}=$ $\left|\phi_{k}-\phi_{|\mathbf{k}+\mathbf{q}|}\right|^{2} / 2$ (where the factor $1 / 2$ avoids double counting).

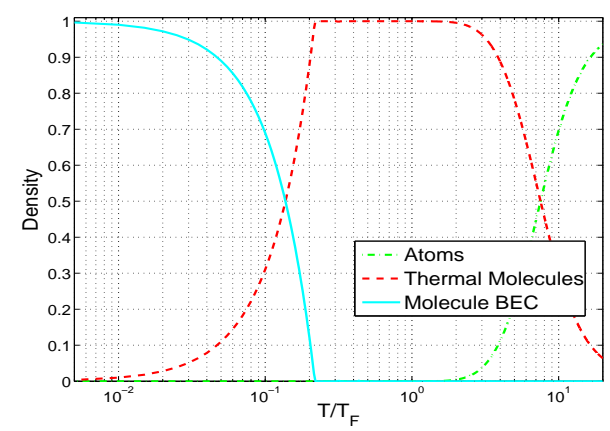

FIG. 4: Contributions to the total atom density (in units of $n$ ) from the molecular BEC, the thermal molecules, and the free atoms.

The BCS wave function correctly describes a condensate of non-overlapping bosons (Cooper pairs) with binding energy $E_{b}=-\hbar^{2} / m a^{2}$ in the BEC limit $a \rightarrow 0^{+}$. To connect this wave function to the above analysis, we note that in the BCS expression the Bragg scattering (5), it is the second term that describes the breaking of Cooper pairs. Thus, BCS theory yields $|M(\mathbf{k}, \mathbf{q})|^{2}=$ $\left(u_{k} v_{\mathbf{k}+\mathbf{q}}-v_{k} u_{\mathbf{k}+\mathbf{q}}\right)^{2} / 2$. In the BEC limit, we have $u_{k} \rightarrow 1$ and $v_{k} \rightarrow \Delta /\left(2 \epsilon_{k}+\left|E_{b}\right|\right)$ with $\Delta \rightarrow 2 \sqrt{\pi n} /(m \sqrt{a})$. BCS theory therefore yields $\phi_{k} \rightarrow v_{k}$ for the Fourier transform of the molecular wave function and

$$
\phi(r)=\sum_{\mathbf{k}} v_{k} e^{i \mathbf{k r}} \propto \frac{e^{-r / a}}{\sqrt{a} r}
$$

in the BEC limit. This is the asymptotic bound state wave function for a potential with scattering length $a$ as expected. We therefore use the BEC limit of the BCS result for the matrix element $|M(\mathbf{k}, \mathbf{q})|^{2}$. Note that we neglect any closed channel components deep in the BEC regime.

\section{A. Results}

The populations of the molecular and atomic states are found by solving the atom-molecule equilibrium problem; the density of the thermal molecules, the molecular BEC and the thermal atoms are shown in Fig. 团for varying $T$.

In Fig. 5. we plot the scattering rate as a function of $\omega$ obtained from (8) for a molecule energy $E_{b}=-22.22 \epsilon_{F}$ (corresponding to $k_{F} a=0.3$ ) and for momentum transfer $q=k_{F}$. The scattering rate is shown in Fig. 5 for a pure molecular BEC at $T=0$ (a) and for thermal molecules at $T=0.5 T_{F}$ above the BEC critical temperature $T_{c}=0.218 T_{F}$. For $T=0$, the rate is given by (10) and the threshold for the scattering is given by $\omega \geq-E_{b}+q^{2} / 4 m$ - the minimum energy required to break a $p=0$ molecule and create two $\downarrow$ atoms with momenta $\mathbf{q} / 2$ and $-\mathbf{q} / 2$. The rate from the thermal molecules at $T=0.5 T_{F}$ obtained from (11) does not differ significantly from that of the molecular BEC 


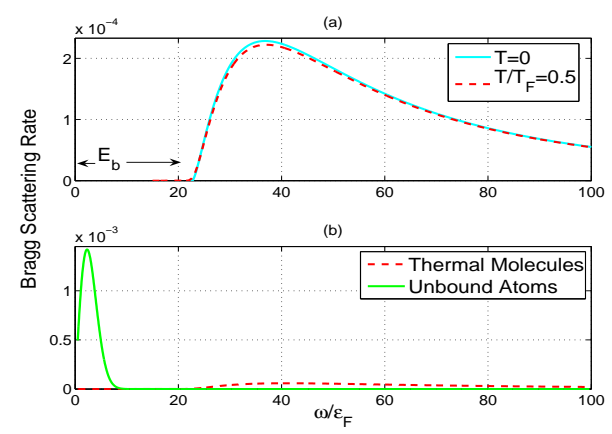

FIG. 5: Bragg scattering rate in units of $\pi \mathcal{I}^{2} n / \epsilon_{F}$ : from the molecular BEC, the thermal molecules, and the free atoms.

at $T=0$. This is because both temperatures are so low that there are essentially no atoms present, as one sees in Fig. [4 The only effect of finite temperature is then a small shift $\omega \geq \min \left(-E_{b}+q^{2} / 4 m-q p / 2 m\right) \sim$ $-E_{b}+q^{2} / 4 m-q \sqrt{k_{B} T / m}$ in the threshold for scattering, from (11). Thus, the onset of Bose-Einstein condensation of molecules has only minor effects on the Bragg scattering rate since $\left|E_{b}\right| \gg k_{B} T_{c}$ in the BEC limit.

In Fig. 罒(b) we plot the scattering rate for $T=2.5 T_{F}$, at which most of the atoms are still bound as molecules but some free atoms are present, as can be seen from Fig. 4 The Bragg scattering consists of a signal down to $\omega=$ 0 from the free atoms, given by (9), and a signal from the thermal molecules for $\omega \gtrsim-E_{b}$, given by (11). Note that even though the atomic signal at small $\omega$ is much larger than the molecular signal at high $\omega$, the contribution to the f-sum rule from the molecules is much larger than that from the free atoms. For higher temperatures, the molecular signal of course disappears with the vanishing molecule density, and the Bragg scattering is given by that of the free atoms only.

\section{STRONG COUPLING UNITARITY LIMIT}

The scattering length diverges, $k_{F}|a| \rightarrow \infty$, at the Feshbach resonance. In this unitarity limit, the interactions are strong and considerably modify the properties of the gas. An important characteristic of the Fermi gas at unitarity is the presence of a pseudogap in the normal phase close to $T_{c}$; i.e., the suppression of the single particle DOS around the Fermi surface [15]. A pseudogap has been observed experimentally for high $T_{c}$ superconductors where it is presently subject to intense investigations [16]. Since a gap in the spectrum of single particle excitations naturally leads to a gap in the low temperature Bragg spectrum (Figs. 35), we now examine whether the pseudogap can be detected in Bragg spectroscopy.

We calculate the Bragg spectrum at unitarity using a minimal many body theory that includes the correct twoparticle physics leading to the resonance. For the single channel problem, such a theory including pair fluctua- tions in the ladder approximation was originally developed many years ago 17 and later examined in great detail by several authors 14, 15. This approach was then extended to the multichannel case relevant for atoms interacting via a Feshbach resonance, treating the bound state as a point boson [18, 19, 20, 21], and taking into account that the bound state is a composite two-fermion object 22.

The atoms are described by the atomic propagator $G(q, z)^{-1}=G_{0}(q, z)^{-1}-\Sigma(q, z)$ with $G_{0}(q, z)^{-1}=z-\xi_{q}$. The atom self-energy is given in the ladder approximation by

$$
\Sigma\left(q, \omega_{n}\right)=\beta^{-1} \operatorname{Tr}\left[\Gamma\left(\mathbf{K}, \mathbf{p}, \mathbf{p}, \omega_{n}+\omega_{m}\right) G_{0}\left(k, \omega_{m}\right)\right]
$$

where $\mathbf{K}=\mathbf{q}+\mathbf{k}, \mathbf{p}=(\mathbf{q}-\mathbf{k}) / 2$, the trace denotes a sum over Matsubara frequencies $\omega_{m}=i(2 m+1) \pi T$ and integration over $\mathbf{k}$, and $\Gamma$ is the many-body scattering matrix depending on the center of mass $\mathbf{K}$ and relative momenta $\mathbf{p}$ of the two scattering particles [10]. In the limit of a broad resonance, the effective interaction mediated by the Feshbach molecule can be regarded as instantaneous with a resonant scattering length $|a| \rightarrow \infty$ at unitarity [21]. Neglecting the frequency and momentum dependence of the effective interaction coming from the molecular state, we recover the single channel theory scattering matrix

$$
\Gamma(K, z)^{-1}=\frac{m}{4 \pi a}-\Pi(K, z) .
$$

The pair propagator is, as usual,

$$
\Pi(K, z)=\int \frac{d^{3} k}{(2 \pi)^{3}}\left(\frac{1-f-f^{\prime}}{z-\xi-\xi^{\prime}}+\frac{m}{q^{2}}\right) .
$$

Here, $\xi=\xi_{\mathbf{K} / 2+\mathbf{k}}, \xi^{\prime}=\xi_{\mathbf{K} / 2-\mathbf{k}}, f=f(\xi)$, and $f^{\prime}=f\left(\xi^{\prime}\right)$. The structure of Eqs. (13)-(15) is indicated in Fig. [6 (a).

To obtain the Bragg spectrum, we need to evaluate the atomic propagator at frequency $\omega+i \eta$. (From here on we do not write the infinitesimal positive imaginary part of the frequency explicitly.) From (13), we obtain

$$
\begin{gathered}
\Sigma(q, \omega)=\int \frac{d^{3} k}{(2 \pi)^{3}} \int_{-\infty}^{\infty} \frac{d \epsilon}{2 \pi}\left[f(\epsilon) A_{0}(k, \epsilon) \Gamma\left(k^{\prime}, \epsilon^{\prime}\right)-\right. \\
\left.n_{B}\left(\epsilon^{\prime}\right) A_{\mathrm{m}}\left(k^{\prime}, \epsilon^{\prime}\right) G_{0}(k, \epsilon)\right] .
\end{gathered}
$$

Here $\mathbf{k}^{\prime}=\mathbf{k}+\mathbf{q}, \quad \epsilon^{\prime}=\epsilon+\omega$, and $A_{0}(k, \omega)=$ $-2 \operatorname{Im} G_{0}(k, \omega)$ and $A_{\mathrm{m}}(k, \omega)=-2 \operatorname{Im} \Gamma(k, \omega)$ are the spectral functions for non-interacting atoms and the pair propagator, respectively. We evaluate the real and imaginary parts of $\Sigma$ directly from (16). We also calculate the real part from the imaginary part using a KramersKronig relation, and find that the two calculations agree to a high accuracy, confirming the consistency of the numerical calculations.

In order to evaluate medium effects, we need to determine $T_{c}$ and the chemical potential, $\mu$. We determine $\mu$ by calculating the thermodynamic potential $\Omega$ at unitarity in the ladder approximation. The correction $\Delta \Omega$ to 
(a)

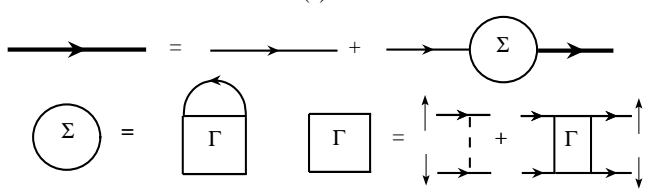

(b)

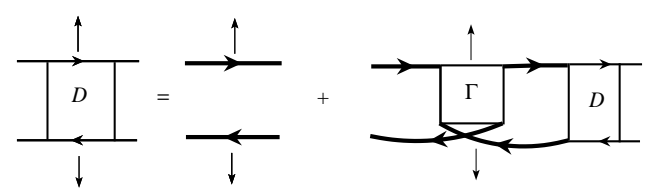

FIG. 6: (a) The atomic propagator in the ladder approximation for a broad resonance where the Feshbach interaction is effectively instantaneous at unitarity; (b) the spin-flip correlation function in a conserving ladder approximation.

the ideal gas result can be expressed as an integral over the phase of the on-shell scattering matrix $\Gamma$ 23]:

$$
\Delta \Omega=\int \frac{d^{3} K d^{3} k}{(2 \pi)^{6}} \frac{f(\xi) f\left(\xi^{\prime}\right)}{\operatorname{Im} \Pi\left(\mathrm{K}, \xi+\xi^{\prime}\right)} \theta\left(K, \xi+\xi^{\prime}\right)
$$

with $\xi, \xi^{\prime}$ defined below (15). The angle $\theta(\omega)$ is defined by writing $\Gamma(\omega)=|\Gamma| e^{i \theta}$. Equation (17) reduces to the virial expansion for $\Omega$ at high temperatures. The chemical potential $\mu$ is then determined by $-\partial \Omega / \partial_{\mu}=n$.

In order to calculate the spin-flip correlation function consistently, one needs in principle to choose a functional $\Phi[G]$, generate the self-energy as $\Sigma=\delta \Phi / \delta G$, and then generate the correlation functions from the kernel $\delta \Sigma / \delta G$ [24]. The diagrams relevant for the ladder approximation are given in Fig. [6] (b) where all propagators are full Green's functions, $G$, including those in $\Gamma$. The conserving spin-flip correlation function thus obeys an integral equation with $\Gamma$ and the full $G$ 's. The imaginary part of the first term in this equation is

$$
\operatorname{Im} \mathcal{D}_{0}(q, \omega)=-\frac{1}{2} \int \frac{d^{3} k}{(2 \pi)^{3}} \int_{-\infty}^{\infty} \frac{d \epsilon}{2 \pi} A A^{\prime}\left(f-f^{\prime}\right)
$$

with $A=A(k, \omega), A^{\prime}=A\left(k^{\prime}, \omega\right)$, and $\mathbf{k}^{\prime}$ and $\xi^{\prime}$ defined as in (16). The spectral function $A(k, \omega)=-2 \operatorname{Im} G(k, \omega)$ describes the excitations of the interacting atoms.

\section{A. Results}

We calculate the Bragg scattering rate at the critical temperature $T_{c}$ where we expect the pseudogap effects to be most pronounced. From the thermodynamic potential (17), we obtain $\mu(T)$ and can calculate the critical temperature from the Thouless criterion $\Gamma^{-1}\left(0,0, T_{c}, \mu\right)=0$. We then evaluate the atom self-energy from (16) and from this obtain the spectral function.

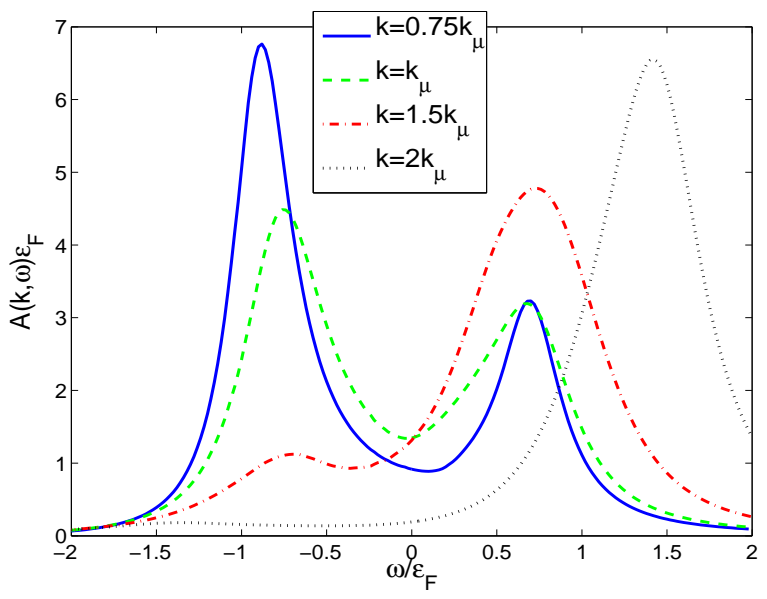

FIG. 7: The atom spectral function $A(k, \omega)$ at unitarity and $T=T_{c}$ for various momenta.
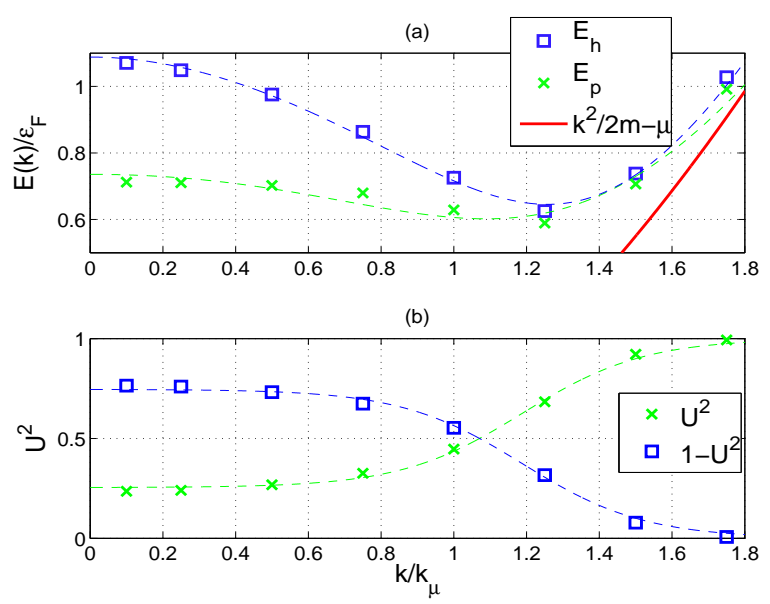

FIG. 8: (a) The positions $E_{\mathrm{h}}(k)$ and $E_{\mathrm{p}}(k)$ of the hole and particle peaks of $A(k, \omega)$ as a function of $k$ at unitarity and for $T=T_{c}$. The solid line is the free particle energy $k^{2} / 2 m-$ $\mu$. (b) The corresponding spectral weights of the particle and hole peaks. The dashed lines are fits to simple analytic functions.

To understand the Bragg scattering rate at unitarity, we first analyze the effects of the interactions on the single particle spectrum, described by the spectral function, $A(k, \omega)$. In Fig. [7] we plot $A(k, \omega)$ at $T_{c}$ for various momenta, measured in units of $k_{\mu}=\sqrt{2 m \mu}$. We have chosen parameters corresponding to a resonant interaction with $\left|k_{\mathrm{F}} a\right|=11.8 \gg 1$ and a negligible effective range. For this set of parameters, we find $T_{c} \approx 0.26 T_{\mathrm{F}}$ with $\mu\left(T_{c}\right) \approx 0.45 \epsilon_{F}$ in good agreement with other BECBCS crossover results based on a similar approximations [18, 25]. The spectral functions obey the sum rule $\int A(\omega) d \omega / 2 \pi=1$ to a very good approximation, providing another important check of the numerical calculations.

In Fig. 7 we clearly see the double peak structure of $A(k, \omega)$ with the spectral weight at $\omega=0$ significantly 


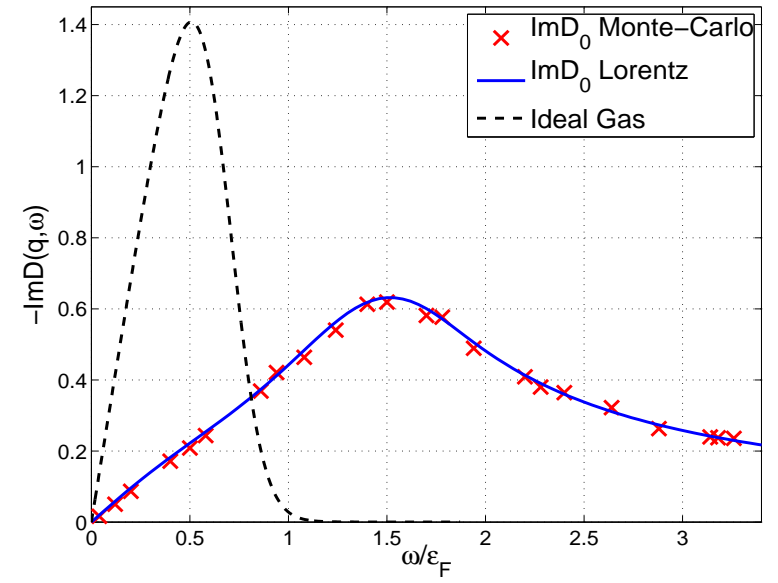

FIG. 9: $-\operatorname{Im} D_{0}(q, \omega)$ at unitarity and $T=T_{c}$ in units of $n / 2 \epsilon_{F}$ as a function of $\omega$ for(color online).

suppressed for all $k$. This suppression is the characteristic feature of the pseudogap. We refer to the peak at $\omega<0$ as the hole peak at frequency $-E_{\mathrm{h}}$, and the peak at $\omega>0$ as the particle peak at $E_{\mathrm{p}}$. The positions of the two peaks found by fitting to a double Lorentzian (normalized such that its total spectral weight is $2 \pi$ ) are plotted in Fig. 8 (a).

We see that the distance of the maxima to $\omega=0$ reaches a minimum: $\min E_{\mathrm{h}}(k) \sim \min E_{\mathrm{p}}(k) \sim 0.6 \epsilon_{F}$ at $k \sim 1.2 k_{\mu}$, providing a qualitative value for the pseudogap. The spectral weight of the hole peak decreases and that of the particle peak increases with increasing $k$. This behavior is illustrated in Fig. 8 (b) where we plot the spectral weights $U^{2}$ of the particle peak and the hole peak $1-U^{2}$ obtained by fitting to the double Lorentzian. For large $k / k_{\mu}$, the spectral function approaches that of an ideal gas with a single peak at $\omega=k^{2} / 2 m-\mu$ as expected. The behavior of the spectral function at unitarity as described here is in general agreement with the analysis of Ref. 15]. The spectral function behaves qualitatively as the BCS spectral function

$$
A_{\mathrm{BCS}}(k, \omega)=v_{k}^{2} 2 \pi \delta\left(\omega+E_{k}\right)+u_{k}^{2} 2 \pi \delta\left(\omega-E_{k}\right),
$$

with two peaks located around $\omega=0$. There are important differences from BCS theory though. The two peaks for the spectral function in the unitarity limit at $T_{c}$ have large widths (which are unequal for the particle and hole peaks) and the spectral density is suppressed but finite at $\omega=0$. The pseudogap is a suppression of spectral density and not a real gap. Also, the positions of the two peaks are not symmetric around $\omega=0$ and the minimum gap is not at $k=k_{\mu}$ but slightly above, as can be seen from Fig. 8

We now ask whether the pseudogap behavior of the spectral function at unitarity has consequences for the Bragg spectrum. In Fig. 9] we plot $\operatorname{Im}_{0}(q, \omega)$ for $q=k_{\mu} / 2$ and $T=T_{c}$. The $\times$ 's are obtained from (18) using the spectral function calculated from (16). We eval- uate the (computationally demanding) multidimensional integrations using a Monte Carlo routine. The solid line in Fig. 9] is obtained by using the double Lorentzian fit $A_{L}(k, \omega)$ for the spectral function in (18). Here, the position of the hole $E_{h}(k)$ and particle $E_{p}(k)$ peaks, the width and the weight $U^{2}(k)$ are calculated as functions of momentum $k$ using approximate functions obtained from fitting $A(k, \omega)$ to the double Lorentzian for various $k$. The approximate functions for $E_{h}(k), E_{p}(k)$, and $U^{2}(k)$ are given as dashed lines in Fig. 8 Once the approximate double Lorentzian form $A_{L}(k, \omega)$ is obtained, the integration in (18) is much faster than when using the spectral function $A(k, \omega)$ obtained from the full ladder calculation. We see from Fig. 9 that the double Lorentzian model $A_{L}(k, \omega)$ yields results for $\operatorname{Im}_{0}(q, \omega)$ in good agreement with the full numerical calculation based on $A(k, \omega)$. From this, we conclude that the pseudogap spectrum close to $T_{c}$ can be well described by a simple double peak spectral function.

Comparing to the ideal gas scattering rate for the same density and temperature, also plotted in Fig. 9 we see that interactions suppress the spectral weight of $\operatorname{Im} \mathcal{D}_{0}(q, \omega)$ at low $\omega$ and push it to higher frequencies. This is a direct effect of the pseudogap. As opposed to the real gap in the $T=0$ Bragg spectrum, the spectral density is however finite at small $\omega$. This is as expected since the single particle spectral density is suppressed but finite for $\omega=0$ (Fig. 77). Also, $T_{c} \simeq 0.26 \epsilon_{F}$ is comparable to the pseudogap $\sim 0.6 \epsilon_{F}$ so thermal excitations are present. From (18), we see that that both effects make $\operatorname{Im} \mathcal{D}_{0}(q, \omega)$ finite for small $\omega$.

The approximation $\mathcal{D}_{0}(q, \omega)$ to the spin-flip correlation function is not conserving and does therefore not obey the f-sum rule. To obtain a conserving approximation, we need to solve the integral equation in Fig. 6](b). This will change the quantitative form for $\mathcal{D}(q, \omega)$. The solution is however an iteration with the $\Gamma$ matrix of $\mathcal{D}_{0}(q, \omega)$. We therefore expect $\operatorname{Im} \mathcal{D}(q, \omega)$ to have the same qualitative characteristics as $\operatorname{Im} \mathcal{D}_{0}(q, \omega)$, i.e., a suppressed but finite spectral weight at low $\omega$. Solving the fully self-consistent equation for the spin-flip correlation function is beyond the scope of this paper 26].

In the BCS limit, we see that the emergence of a gap in the single particle spectrum leads to a maximum in the Bragg scattering rate just below $T_{c}$ (Fig. 2). One could therefore expect a similar maximum to appear at unitarity at a temperature $T^{*}$ defined as the temperature where the pseudogap emerges. However, the presence of a maximum requires the spectral function to have welldefined sharp quasiparticle peaks like in the BCS case (19). The pseudogap spectral function at unitarity (Fig. (7) on the other hand has peaks of width $\sim \mathcal{O}\left(\epsilon_{F}\right)$. There will therefore be no maximum in the Bragg scattering rate for $T \sim T^{*}$ due to the emergence of the pseudogap [27].

We conclude that the pseudogap present in the normal phase close to $T_{c}$ at unitarity leads to a significant suppression of the low frequency Bragg scattering rate. The 
rate remains however finite since the pseudogap is only a suppression of the single particle spectral density, and because $T_{c}$ is comparable to the value of the pseudogap. The emergence of a pseudogap with lowering $T$ does not give rise to a maximum in the scattering rate, as opposed to the BCS case. This is because the quasiparticles are strongly damped at unitarity.

\section{EXPERIMENTS}

We briefly comment on the possible experimental observation of the effects described in this paper. A typical Fermi energy for trapped atomic gases is $\sim \mathcal{O}\left(10^{4}\right) \mathrm{Hz}$. Thus, we require a frequency resolution of the Bragg experiment $\ll 10^{4} \mathrm{~Hz}$. Likewise, a typical Fermi momentum of the gas is $k_{F} \sim \mathcal{O}\left(10^{6}\right) \mathrm{m}^{-1}$ which is the same order of magnitude as the wave numbers for optical lasers. This means that the resulting angle between two laser beams needed to obtain a momentum transfer of $\sim k_{F}$ is reasonable. The results presented in this paper are for a homogenous system. From the Thomas-Fermi approximation, we expect the presence of the trapping potential to smooth out features like the gap in the Bragg spectrum. To avoid such smoothening, one could focus the laser beams on a smaller section of the cloud where the gas can be regarded as homogeneous. In this case, the results presented in the present paper become directly applicable.

\section{ACKNOWLEDGMENTS}

We are grateful to Cindy Regal and Chris Pethick for helpful discussions. We thank the Aspen Center for Physics where parts of this research were carried out. This research was also supported in part by NSF Grants PHY03-55014 and PHY05-00914.
[1] D. M. Stamper-Kurn, A. P. Chikkatur, A. Görlitz, S. Inouye, S. Gupta, D. E. Pritchard, and W. Ketterle, Phys. Rev. Lett. 83, 2876 (1999); J. Steinhauer, R. Ozeri, N. Katz, and N. Davidson, Phys. Rev. Lett. 88, 120407 (2002).

[2] G. M. Bruun and G. Baym, Phys. Rev. Lett. 93, 150403 (2004).

[3] L. C. Hebel and C. P. Slichter, Phys. Rev. 113, 1504 (1959); L. C. Hebel, Phys. Rev. 116, 79 (1959).

[4] C. Chin, M. Bartenstein, A. Altmeyer, S. Riedl, S. Jochim, J. Hecker Denschlag, and R. Grimm, Science 305, 1128 (2004).

[5] Z. Yu and G. Baym, Phys. Rev. A 73, 063601 (2006).

[6] H. P. Büchler, P. Zoller, and W. Zwerger, Phys. Rev. Lett. 93, 080401 (2004).

[7] B. Deb, J. Phys. B 39, 529 (2006).

[8] B. Mihaila, S. Gaudio, K. B. Blagoev, A. V. Balatsky, P. B. Littlewood, and D. L. Smith, Phys. Rev. Lett. 95, 090402 (2005).

[9] See e.g. I. Carusotto, J. Phys. B 39, S211 (2006).

[10] A. L. Fetter and J. D. Walecka, Quantum Theory of Many-Particle Systems, (McGraw-Hill, 1971).

[11] J. W. Negele and H. Orland, Quantum Many-Particle Systems, (Addison-Wesley, 1988).

[12] A. J. Leggett, Phys. Rev. 140 A1869 (1965); ibid, 147 119 (1966).

[13] G. M. Bruun and B. R. Mottelson, Phys. Rev. Lett. 87, 270403 (2001).

[14] See M. Randeria in Bose-Einstein Condensation, edited by A. Griffin et al. (Cambridge University Press, Cambridge, 1995) and references therein.

[15] A. Perali, P. Pieri, G. C. Strinati, and C. Castellani,
Phys. Rev. B 66, 024510 (2002).

[16] M. R. Norman and C. Pépin, Rep. Prog. Phys. 66, 1547 (2003).

[17] P. Nozières and S. Schmitt-Rink, J. Low Temp. Phys. 59, 195 (1985).

[18] M. Holland, S. J. J. M. Kokkelmans, M. L. Chiofalo, and R. Walser, Phys. Rev. Lett. 87, 120406 (2001); J. N. Milstein, S. J. J. M. F. Kokkelmans, and M. J. Holland, Phys. Rev. A 66, 043604 (2002).

[19] Y. Ohashi and A. Griffin, Phys. Rev. A 67, 033603 (2003).

[20] R. Duine and H. T. C. Stoof, J. Opt. B 5, S212 (2003).

[21] G. M. Bruun and C. J. Pethick, Phys. Rev. Lett. 92, 140404 (2004).

[22] G. M. Bruun, A. D. Jackson, and E. E. Kolomeitsev, Phys. Rev. A 71, 052713 (2005).

[23] G. M. Bruun, Phys. Rev. A 70, 053602 (2004).

[24] G. Baym and L. P. Kadanoff, Phys. Rev. 124, 287 (1961); G. Baym, ibid 127, 1391 (1962).

[25] P. Pieri, L. Pisani, and G. C. Strinati, Phys. Rev. Lett. 92, 220404 (2004).

[26] A possible approach is via quantum Monte Carlo. See, e.g., calculations of the spin susceptibility in the pseudogap regime of the BCS-BEC crossover by M. Randeria, N. Trivedi, A. Moreo, and R. Scalettar, Phys. Rev. Lett. 69, 2001 (1992), and N. Trivedi and M. Randeria, Phys. Rev. Lett. 75, 312 (1995).

[27] Similarly, the broadening of the quasiparticle levels in the cuprates is likely responsible for the absence there of a Hebel-Slichter peak. C. Slichter, private communication. 